\title{
Crescimento e trocas gasosas do feijão-fava submetido à adubação e condicionadores
}

\section{do solo}

\author{
Initial growth and gas exchange of fava beans submitted to mineral manure and soil conditioners \\ Crecimiento e intercambio de gases de habas sometidas a fertilización y acondicionadores del suelo
}

Recebido: 16/06/2021 | Revisado: 24/06/2021 | Aceito: 26/06/2021 | Publicado: 11/07/2021

Anny Karolinny de França Soares ORCID: https://orcid.org/0000-0001-8638-0806 Universidade Federal de Campina Grande, Brasil E-mail: anny.karolinny@ hotmail.com

Karla da Nóbrega Gomes ORCID: https://orcid.org/0000-0001-9430-4176 Universidade Federal de Campina Grande, Brasil E-mail: karlindagomes@hotmail.com

Décio Carvalho Lima ORCID: https://orcid.org/0000-0001-5466-9832 Universidade Federal de Campina Grande, Brasil E-mail: decio.lima@gmail.com

Luderlandio Andrade Silva

ORCID: https://orcid.org/0000-0001-9496-5820

Universidade Federal de Campina Grande, Brasil E-mail: luderlandioandrade@gmail.com Lauter Silva Souto

ORCID: https://orcid.org/0000-0002-1053-0297 Universidade Federal de Campina Grande, Brasil E-mail: lauter@ccta.ufcg.edu.br

João de Andrade Dutra Filho ORCID: https://orcid.org/0000-0002-9515-7267 Universidade Federal de Pernambuco, Brasil E-mail: joaodutrafilho7@gmail.com Rômulo Gil de Luna

ORCID: https://orcid.org/0000-0002-8350-5615 Universidade Federal de Campina Grande, Brasil E-mail: romulo.gil.luna@gmail.com

Anielson dos Santos Souza

ORCID: https://orcid.org/0000-0003-0145-0989 Universidade Federal de Campina Grande, Brasil E-mail: anielson@ccta.ufcg.edu.br

Rosilene Agra da Silva ORCID: https://orcid.org/0000-0001-9232-7403 Universidade Federal de Campina Grande, Brasil E-mail: rosileneagra@hotmail.com

Jacob Silva Souto

ORCID: https://orcid.org/0000-0001-7619-0400 Universidade Federal de Campina Grande, Brasil E-mail: jacob_souto@yahoo.com.br

\section{Resumo}

A cultura do feijão-fava, apesar do cultivo um tanto restrito, apresenta grande importância socioeconômica para o estado da Paraíba, devido sua adaptabilidade às condições ideais de clima e solo. Desse modo, essa pesquisa teve por objetivo avaliar o crescimento inicial e fisiologia de acessos de feijão-fava (Phaseolus lunatus L.) submetidos à adubação mineral e condicionadores de solo nas condições de Pombal-PB. O delineamento experimental utilizado foi em blocos casualizados com seis repetições, em esquema fatorial $10 \mathrm{x} 2$, com dois genótipos de fava (Luzente e Venturinha) e dez tratamentos. As plantas foram submetidas aos seguintes tratamentos: $\mathrm{T}_{1}=$ Controle; $\mathrm{T}_{2}=\mathrm{NPK}_{100 \%}$; $\mathrm{T}_{3}=\mathrm{N}_{\text {Barvar }}+\mathrm{PK}_{100 \%} ; \mathrm{T}_{4}=\mathrm{N}_{\text {Barvar }}+\mathrm{PK}_{100 \%}+\mathrm{N}_{50 \%} ; \mathrm{T}_{5}=\mathrm{K}_{\text {Barvar }}+\mathrm{NP}_{100 \%} ; \mathrm{T}_{6}=\mathrm{K}_{\text {Barvar }}+\mathrm{NP}_{100 \%}+\mathrm{K}_{50 \%} ; \mathrm{T}_{7}=\mathrm{P}_{\mathrm{Barvar}}+$ $\mathrm{NK}_{100 \%} ; \mathrm{T}_{8}=\mathrm{P}_{\text {Barvar }}+\mathrm{NK}_{100 \%}+\mathrm{P}_{50 \%} ; \mathrm{T}_{9}=\mathrm{N}_{\text {Barvar }}+\mathrm{P}_{\text {Barvar }}+\mathrm{K}_{\text {Barvar }} ; \mathrm{T}_{10}=\mathrm{N}_{\text {Barvar }}+\mathrm{P}_{\text {Barvar }}+\mathrm{K}_{\text {Barvar }}+\mathrm{NPK}_{50 \%}$. Foram avaliados as variáveis de crescimento diâmetro de caule e número de folhas e; as relativas as trocas gasosas taxa de assimilação de $\mathrm{CO}_{2}$, transpiração, condutância estomática, concentração interna de $\mathrm{CO}_{2}$, eficiência instantânea do uso da água e eficiência instantânea da carboxilação. Os maiores valores obtidos para diâmetro de caule e melhores respostas aos parâmetros fisiológicos foram observados nos tratamentos em que se utilizaram os condicionadores de 
solo via sementes e fertirrigação. A adubação mineral NPK associada aos condicionadores de solo podem compor o manejo nutricional da cultura do feijão-fava nas condições em que foram desenvolvidas o estudo.

Palavras-chave: Fabaceae; Phaseolus lunatus L.; Fertilização; Agricultura familiar.

\begin{abstract}
The fava beans crop, despite the somewhat restricted cultivation, has great socioeconomic importance for the state of Paraiba, due to its adaptability to ideal climate and soil conditions. Thus, this research aimed to evaluate the initial growth and physiology of fava beans accessions (Phaseolus lunatus L.) subjected to mineral fertilization and soil conditioners under Pombal-PB conditions. The experimental design used was a randomized block design with six repetitions, in a $10 \times 2$ factorial scheme, with two broad beans genotypes (Luzente and Venturinha) and ten treatments. The plants were subjected to the following treatments: $\mathrm{T}_{1}=$ control; $\mathrm{T}_{2}=\mathrm{NPK}_{100 \%} ; \mathrm{T}_{3}=\mathrm{N}_{\mathrm{Barvar}}+\mathrm{PK}_{100 \%}$; $\mathrm{T}_{4}=\mathrm{N}_{\text {Barvar }} \mathrm{r}+\mathrm{PK}_{100 \%}+\mathrm{N}_{50 \%} ; \mathrm{T}_{5}=\mathrm{K}_{\text {Barvar }}+\mathrm{NP}_{100 \%} ; \mathrm{T}_{6}=\mathrm{K}_{\text {Barvar }}+\mathrm{NP}_{100 \%}+\mathrm{K}_{50 \%} 0 \% ; \mathrm{T}_{7}=\mathrm{P}_{\text {Barvar }}+\mathrm{NK}_{100 \%} ; \mathrm{T}_{8}=\mathrm{P}_{\text {Barvar }}$ $+\mathrm{NK}_{100 \%}+\mathrm{P}_{50 \%} ; \mathrm{T}_{9}=\mathrm{N}_{\text {Barvar }}+\mathrm{P}_{\text {Barvar }}+\mathrm{K}_{\text {Barvar; }} ; \mathrm{T}_{10}=\mathrm{N}_{\text {Barvar }}+\mathrm{P}_{\text {Barvar }}+\mathrm{K}_{\text {Barvar }}+\mathrm{NPK}_{50 \%}$. The growth variables stem diameter and number of leaves were evaluated, as well as the gas exchange variables $\mathrm{CO}_{2}$ assimilation rate, transpiration, stomatal conductance, internal $\mathrm{CO}_{2}$ concentration, instantaneous water use efficiency and instantaneous carboxylation efficiency. The highest values obtained for stem diameter and better responses to physiological parameters were observed in treatments that used soil conditioners via seeds and fertigation. The mineral NPK fertilization associated with soil conditioners can compose the nutritional management of the fava beans crop under the conditions in which the study was developed.
\end{abstract}

Keywords: Fabaceae; Phaseolus lunatus L.; Fertilization; Family farming.

\title{
Resumen
}

El cultivo de habas, a pesar de su cultivo algo restringido, tiene una gran importancia socioeconómica para el estado de Paraíba, debido a su adaptabilidad a las condiciones ideales de clima y suelo. Así, esta investigación tuvo como objetivo evaluar el crecimiento inicial y fisiología de accesiones de habas (Phaseolus lunatus L.) sometidas a fertilización mineral y acondicionadores de suelo en las condiciones de Pombal-PB. El diseño experimental utilizado fue un diseño de bloques al azar con seis repeticiones, en un esquema factorial de $10 \times 2$, con dos genotipos de haba (Luzente y Venturinha) y diez tratamientos. Las plantas fueron sometidas a los siguientes tratamientos: $\mathrm{T}_{1}=$ Controle; $\mathrm{T}_{2}=\mathrm{NPK}_{100 \%} ; \mathrm{T}_{3}=\mathrm{N}_{\text {Barvar }}+\mathrm{PK}_{100 \%} ; \mathrm{T}_{4}=\mathrm{N}_{\text {Barvar }}+\mathrm{PK}_{100 \%}+\mathrm{N}_{50 \%} ; \mathrm{T}_{5}=\mathrm{K}_{\text {Barvar }}+\mathrm{NP}_{100 \%} ; \mathrm{T}_{6}=\mathrm{K}_{\text {Barvar }}+\mathrm{NP}_{100 \%}+\mathrm{K}_{50 \%} ;$ $\mathrm{T}_{7}=\mathrm{P}_{\text {Barvar }}+\mathrm{NK}_{100 \%} ; \mathrm{T}_{8}=\mathrm{P}_{\text {Barvar }}+\mathrm{NK}_{100 \%}+\mathrm{P}_{50 \%} ; \mathrm{T}_{9}=\mathrm{N}_{\text {Barvar }}+\mathrm{P}_{\text {Barvar }}+\mathrm{K}_{\text {Barvar }} ; \mathrm{T}_{10}=\mathrm{N}_{\text {Barvar }}+\mathrm{P}_{\text {Barvar }}+\mathrm{K}_{\text {Barvar }}+$ $\mathrm{NPK}_{50 \%}$. Las variables de crecimiento diámetro del tallo y número de hojas y; los relacionados con el intercambio de gases, la tasa de asimilación de $\mathrm{CO}_{2}$, la transpiración, la conductancia estomática, la concentración interna de $\mathrm{CO}_{2}$, la eficiencia del uso instantáneo del água y la eficiencia de la carboxilación instantánea. Los mayores valores obtenidos para diámetro de tallo y mejores respuestas a parámetros fisiológicos se observaron en tratamientos en los que se utilizaron acondicionadores de suelo vía semillas y fertirrigación. La fertilización mineral NPK asociada con acondicionadores del suelo puede componer el manejo nutricional del cultivo de habas en las condiciones en las que se desarrolló el estudio.

Palabras clave: Fabaceae; Phaseolus lunatus L.; Fertilización; Agricultura familiar.

\section{Introdução}

A cultura do feijão-fava (Phaseolus lunatus L.), também conhecida por feijão-de-lima, fava-de-lima ou simplesmente fava, é uma das alternativas de renda e alimento para a agricultura familiar da região nordeste do Brasil. Nessa região, a fava é consumida sob a forma de grãos maduros, verdes e secos (Silva et. al., 2015). Consiste em uma das quatro espécies do gênero Phaseolus exploradas comercialmente, sendo considerada a segunda espécie mais importante economicamente, superada apenas pelo feijão-comum (Oliveira et. al., 2011).

Embora o estado da Paraíba seja um dos maiores produtores de feijão-fava do país, tem sido notada a diminuição nos índices de produtividade, que pode ser consequência de problemas que vão desde os climáticos aos de fertilidade do solo (Barbosa \& Arriel, 2018). No Brasil, a cultura é plantada principalmente na região Nordeste, Minas Gerais e Rio Grande do Sul, com área colhida de aproximadamente de 36.061 ha (IBGE, 2018). Os principais estados produtores da região Nordeste são: Ceará, Paraíba, Piauí, Pernambuco e Rio Grande do Norte, com área plantada de 9.037, 8.615, 2.211, 1.882, 1.488 ha e uma produção de 3.596, 3.352, 801, 606,419 t ha-1, respectivamente (IBGE, 2018).

Em relação ao manejo nutricional, o nitrogênio é o nutriente mais exigido pelas culturas (Troeh et. al., 2007), o que torna a adubação nitrogenada essencial para que o alcance de uma boa produtividade. A quantidade varia em virtude da 
produtividade desejada, manejo da cultura, condições de solo e clima local (Cavalcante 2017). O fósforo promove o aumento da produção de matéria seca da parte aérea, o incremento do número de vagens e da massa de grãos (Fageria et. al., 2003). A adubação fosfatada também aumenta o teor e acúmulo de P na matéria seca da parte aérea e nos grãos (Fonseca et. al., 2010). Outro elemental vital é o potássio que desempenha um papel importante na tolerância ao estresse hídrico (Zörb et. al., 2014) e está envolvido diretamente no processo de fotossíntese, transporte de assimilados, ativação enzimática e estresse oxidativo (Cakmak 2014, Pettigrew, 2008). A deficiência deste elemento no solo pode impedir o mecanismo de fixação biológica de nitrogênio (N2) pelas leguminosas e afeta o teor de proteína nas sementes (Römheld \& Kirkby, 2010, Taha et. al., 2016).

Nesse contexto, o uso de condicionadores de solo que promovam melhorias nos atributos físicos, químicos e biológicos do solo pode aumentar a disponibilidade de nutrientes e o desenvolvimento do sistema radicular das culturas. $\mathrm{O}$ biofertilizante é composto de microrganismos benéficos que, em função da sua composição, favorece a fixação biológica de nitrogênio e solubilização de íons fosfato e potássio para absorção pelas plantas (Gyaneshwar et. al., 2002; Canuto et. al., 2003). A capacidade de associação de bactérias com plantas leguminosas, por meio do processo de inoculação, ocasiona em uma potencialização da fixação biológica de N, reduzindo a necessidade de adubação mineral e reduz a poluição ambiental. A fixação biológica pode ser responsável por aproximadamente 65\% do total de $\mathrm{N}$ fixado na Terra (Azevedo 2010), sendo o segundo processo biológico mais importante depois da fotossíntese (Moreira \& Siqueira, 2006, Taiz et. al, 2017).

O plantio de acessos de feijão-fava tradicionais com pouca capacidade produtiva, e a inexistência de um manejo adequado da fertilidade do solo tem contribuído, entre outros fatores, para o baixo rendimento e retorno econômico, tornando a fava praticamente uma cultura de subsistência (Oliveira et. al. 2010; Alves et. al., 2008). O uso da adubação mineral e de condicionadores de solo na cultura do feijão-fava na região semiárida ainda apresentam poucos resultados de pesquisa na área. Diante do expoto, o presente trabalho teve como objetivo avaliar o crescimento inicial e as trocas gasosas de acessos de Phaseolus lunatus L. submetido à adubação NPK e condicionadores de solo no semiárido do Estado da Paraíba.

\section{Metodologia}

O trabalho foi conduzido em condições de túnel plástico na Universidade Federal de Campina Grande (UFCG), no

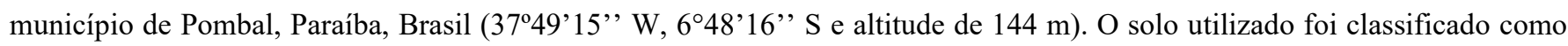
Neossolo Flúvico (Santos et. al., 2018) e Entisol (Soil Survey Staff, 2014), e apresentava as seguintes características químicas: $\mathrm{pH}\left(\mathrm{H}_{2} \mathrm{O}\right)=7,9 ; \mathrm{P}=117 \mathrm{mg} \mathrm{dm}^{-3} ; \mathrm{K}=0,41 \mathrm{cmol}_{\mathrm{c}} \mathrm{dm}^{-3} ; \mathrm{Na}=0,65 \mathrm{cmol}_{\mathrm{c}} \mathrm{dm}^{-3} ; \mathrm{Ca}=3,9 \mathrm{cmol}_{\mathrm{c}} \mathrm{dm}^{-3} ; \mathrm{Mg}=1,5 \mathrm{cmol}_{\mathrm{c}} \mathrm{dm}^{-3} ; \mathrm{CTC}$ $=6,5 \mathrm{cmol}_{\mathrm{c}} \mathrm{dm}^{-3} \mathrm{e}$ matéria orgânica $=31,95 \mathrm{~g} \mathrm{~kg}^{-1}$.

O delineamento experimental utilizado foi o de blocos ao acaso, em esquema fatorial $10 \times 2$, com seis repetições, cujos tratamentos consistiram da combinação de N, P e K (Controle; $\mathrm{NPK}_{100 \%} ; \mathrm{PK}_{100 \%} ; \mathrm{PK}_{100 \%}+\mathrm{N}_{50 \%} ; \mathrm{NP}_{100 \%} ; \mathrm{NP}_{100 \%}+\mathrm{K}_{50 \%}$; $\mathrm{NK}_{100 \%} ; \mathrm{NK}_{100 \%}+\mathrm{P}_{50 \%}$; $\mathrm{NPK}_{50 \%}$ ) associados a três condicionadores de solo ( $\mathrm{N}_{\text {Barvar, }}$, $\mathrm{P}_{\mathrm{Barvar}}$ e $\mathrm{K}_{\mathrm{Barvar}}$ ) em dois acessos de feijãofava $\left(A_{1}=\right.$ Luzente e $A_{2}=$ Venturinha). Os tratamentos consistiram das seguintes combinações: $T_{1}=$ Controle $+A_{1} ; T_{2}=$ Controle $+\mathrm{A}_{2} ; \mathrm{T}_{3}=\mathrm{NPK}_{100 \%}+\mathrm{A}_{1} ; \mathrm{T}_{4}=\mathrm{NPK}_{100 \%}+\mathrm{A}_{2} ; \mathrm{T}_{5}=\mathrm{N}_{\text {Barvar }}+\mathrm{PK}_{100 \%}+\mathrm{A}_{1} ; \mathrm{T}_{6}=\mathrm{N}_{\text {Barvar }}+\mathrm{PK}_{100 \%}+\mathrm{A}_{2} ; \mathrm{T}_{7}=\mathrm{N}_{\mathrm{Barvar}}+$ $\mathrm{PK}_{100 \%}+\mathrm{N}_{50 \%}+\mathrm{A}_{1} ; \mathrm{T}_{8}=\mathrm{N}_{\text {Barvar }}+\mathrm{PK}_{100 \%}+\mathrm{N}_{50 \%}+\mathrm{A}_{2} ; \mathrm{T}_{9}=\mathrm{K}_{\text {Barvar }}+\mathrm{NP}_{100 \%}+\mathrm{A}_{1} ; \mathrm{T}_{10}=\mathrm{K}_{\text {Barvar }}+\mathrm{NP}_{100 \%}+\mathrm{A}_{2} ; \mathrm{T}_{11}=\mathrm{K}_{\text {Barvar }}+$ $\mathrm{NP}_{100 \%}+\mathrm{K}_{50 \%}+\mathrm{A}_{1} ; \mathrm{T}_{12}=\mathrm{K}_{\text {Barvar }}+\mathrm{NP}_{100 \%}+\mathrm{K}_{50 \%}+\mathrm{A}_{2} ; \mathrm{T}_{13}=\mathrm{P}_{\text {Barvar }}+\mathrm{NK}_{100 \%}+\mathrm{A}_{1} ; \mathrm{T}_{14}=\mathrm{P}_{\text {Barvar }}+\mathrm{KN}_{100 \%}+\mathrm{A}_{2} ; \mathrm{T}_{15}=\mathrm{P}_{\text {Barvar }}+$ $\mathrm{NK}_{100 \%}+\mathrm{P}_{50 \%}+\mathrm{A}_{1} ; \mathrm{T}_{16}=\mathrm{P}_{\text {Barvar }}+\mathrm{KN}_{100 \%}+\mathrm{P}_{50 \%}+\mathrm{A}_{2} ; \mathrm{T}_{17}=\mathrm{N}_{\text {Barvar }}+\mathrm{P}_{\text {Barvar }}+\mathrm{K}_{\text {Barvar }}+\mathrm{A}_{1} ; \mathrm{T}_{18}=\mathrm{N}_{\text {Barvar }}+\mathrm{P}_{\text {Barvar }}+\mathrm{K}_{\text {Barvar }}+$ $\mathrm{A}_{2} ; \mathrm{T}_{19}=\mathrm{N}_{\text {Barvar }}+\mathrm{P}_{\text {Barvar }}+\mathrm{K}_{\text {Barvar }}+\mathrm{NPK}_{50 \%}+\mathrm{A}_{1} ; \mathrm{T}_{20}=\mathrm{N}_{\text {Barvar }}+\mathrm{P}_{\text {Barvar }}+\mathrm{K}_{\text {Barvar }}+\mathrm{NPK}_{50 \%}+\mathrm{A}_{2}$.

Utilizou-se unidades experimentais com capacidade de $35 \mathrm{dm}^{3}$, colocando-se em sua parte inferior 2 litros de areia e brita, para evitar entupimento dos drenos. O substrato utilizado foi composto de 8 litros de esterco de curral curtido com 25 litros de solo. 
Os condicionadores de solo na forma de pó utilizados ( $\mathrm{N}_{\text {Barvar, }}, \mathrm{P}_{\text {Barvar }}$ e $\mathrm{K}_{\mathrm{Barvar}}$ da Green BioTech Brasil) foram colocados em copos descartáveis separadamente de acordo com cada tratamento e foi executada sua diluição em $50 \mathrm{ml}$ de água destilada. Em seguida, acrescentaram-se as sementes e aguardou-se 10 minutos que é recomendado pelo fabricante para poder proceder a semeadura assim sendo a primeira aplicaçao do condicionador. Foram semeadas quatro sementes por vaso, sendo realizado o raleio oito dias após a semeadura, deixando-se duas plântulas por vaso. A segunda aplicação dos condicionadores do solo foi realizada via fertirrigação aos 40 dias após a semeadura.

O N $\mathrm{N}_{\text {Barvar }}$, condicionador contendo nitrogênio, contém a estirpe de Azotobacter vinelandii in vivo em $10^{7}$ a $10^{8}$ UFC por grama. $\mathrm{O} \mathrm{P}_{\text {Barvar }}$ contém dois tipos de bactérias solubilizantes de fosfato (PSB) altamente eficientes que secretam ácidos orgânicos e enzimas fosfatase que hidrolisam compostos de fosfato inorgânicos e orgânicos insolúveis em íons fosfato solúvel em torno de raízes, a saber: 1) Pantoea agglomerans, linhagem P5 que libera fosfato forma compostos inorgânicos principalmente pela produção de ácidos orgânicos e; 2) Pseudomonas putida, estirpe P13 que libera fosfato a partir de compostos orgânicos principalmente por segregar enzimas fosfatase. $\mathrm{O}$ condicionador $\mathrm{K}_{\mathrm{Barvar}}$ contém dois tipos de bactérias solubilizantes de potássio, a saber: 1) Pseudomonas vancouverensis Strain, $10^{7}-10^{8}$ UFC/gr e; 2) Pseudomonas koreensis Strain, 107-108 UFC/gr. São embalados como bactérias vivas em $10^{7}-10^{8} \mathrm{CFU}$ por grama ou ml e formulados para ter uma vida de prateleiras por pelo menos seis meses. Estas cepas bacterianas são ativas em alto teor de sal (até 5\%), ampla faixa de pH (5 a 9) e temperatura $\left(10\right.$ a $\left.40^{\circ} \mathrm{C}\right)$. As bactérias hidrolisam compostos insolúveis de potássio no solo ao redor das raízes, liberando o íon para absorção.

A fertilização mineral teve seu início 15 dias após a semeadura, onde foi utilizado como fonte de $\mathrm{N}$ a Uréia (44\% de $\mathrm{N})$; como fonte de potássio o cloreto de potássio $\left(60 \%\right.$ de $\left.\mathrm{K}_{2} \mathrm{O}\right)$ e como fonte de fósforo foi utilizado o Fosfato Monoamônico (MAP) e o Fosfato Monopotássico (MKP), visto que o MAP também é uma fonte de nitrogênio e o MKP é fonte de potássio. Nos tratamentos $T_{5}, T_{6}, T_{7}, T_{8}$ foram utilizados o MKP para que não houvesse uma camuflagem nos resultados, pois nesses tratamentos o condicionador usado foi com bactérias nitrogenadas, já nos tratamentos $\mathrm{T}_{9}, \mathrm{~T}_{10}, \mathrm{~T}_{11}, \mathrm{~T}_{12}$, utilizou o MAP com o mesmo objetivo de não camuflar os resultados, sendo o condicionador com bactérias potássicas. Para os cálculos foram considerados as quantidades de nitrogênio e potássio dos adubos MAP e MKP e as demais adubações foram realizadas semanalmente de acordo com a recomendação de Trani et al. (2015) para a cultura, sendo utilizado durante o experimento 100 $\mathrm{kg}$ de $\mathrm{N}, 60 \mathrm{~kg}$ de $\mathrm{P}_{2} \mathrm{O}_{5}$ e $40 \mathrm{~kg}$ de $\mathrm{K}_{2} \mathrm{O}$.

As plantas foram conduzidas em espaldeira vertical, com arames do tipo $12 \mathrm{~mm}$, possuindo altura de dois metros, sendo colocado um barbante do vaso até o arame, para servir de sustentação para as plantas. Os tratamentos fitossanitários foram feitos conforme a necessidade da cultura.

As irrigações foram realizadas diariamente, de modo a deixar o solo com umidade próxima à máxima capacidade de retenção, com base no método da lisimetria de drenagem, sendo a lâmina aplicada acrescida de uma fração de lixiviação de 20\%. O volume aplicado $\left(\mathrm{V}_{\mathrm{a}}\right)$ por recipiente foi obtido pela diferença entre o volume anterior $\left(\mathrm{V}_{\text {ant }}\right)$ aplicada menos a média de drenagem (d), dividido pelo número de recipientes (n), como indicado na equação 1:

$$
V_{a}=\frac{V_{a n t}-D}{n(1-F L)}
$$

Para o monitoramento dos aspectos morfológicos da cultura, foi realizada análise de crescimento das plantas aos 60 DAS, avaliando-se o número de folhas (NF), a partir da contagem das folhas maduras; diâmetro de caule (DC), medindo-se na base do caule a uma altura de $1 \mathrm{~cm}$ do solo, expresso em $\mathrm{mm}$.

Para as determinações das variáveis fisiológicas da cultura utilizou-se o analisador de gás no infravermelho (IRGA) (LCpro+) com luz constante de $1.200 \mu \mathrm{mol}$ de fótons $\mathrm{m}^{-2} \mathrm{~s}^{-1} \mathrm{e} \mathrm{CO}_{2}$ proveniente do ambiente a uma altura de $3 \mathrm{~m}$ da superfície 
do solo, obtendo-se as seguintes variáveis: i) Assimilação líquida de $\mathrm{CO}_{2}(A)\left(\mu \mathrm{mol} \mathrm{m} \mathrm{m}^{-2} \mathrm{~s}^{-1}\right)$, ii) Transpiração $(E)\left(\mathrm{mol}\right.$ de $\mathrm{H}_{2} \mathrm{O}$ $\left.\mathrm{m}^{-2} \mathrm{~s}^{-1}\right)$, iii) Condutância estomática $(g s)\left(\right.$ mol de $\left.\mathrm{H}_{2} \mathrm{O} \mathrm{m} \mathrm{m}^{-2} \mathrm{~s}^{-1}\right) \mathrm{e}$, iv) Concentração interna de $\mathrm{CO}_{2}(C i)$, na terceira folha contada a partir do ápice. De posse desses dados, foram quantificadas a eficiência instantânea no uso da água $(E U A)(A / E)$ $\left[\left(\mu \mathrm{mol} \mathrm{m}{ }^{-2} \mathrm{~s}^{-1}\right)\left(\mathrm{mol} \mathrm{H}_{2} \mathrm{O} \mathrm{m}^{-2} \mathrm{~s}^{-1}\right)^{-1}\right] \mathrm{e}$ a eficiência instantânea da carboxilação (EiCi) $(\mathrm{A} / \mathrm{Ci})$.

Avaliaram-se os dados obtidos mediante análise de variância pelo teste $\mathrm{F}$ e, nos casos de significância, foi realizado o teste de agrupamento de médias (Scott-Knott a 5\% de probabilidade) para as combinações de NPK (\%) e condicionadores de solo, em cada acesso estudado pelo software SISVAR-ESAL (Ferreira 2011).

\section{Resultados e Discussão}

Na Tabela 1 encontram-se os resultados da análise de variância para o crescimento da cultura do feijão-fava. Observase diferença significativa para o efeito isolado de condicionadores de solo (CON) e para o fator acessos de feijão-fava (AFV) apenas para a variável diâmetro de caule (DC). Não observou-se significância para a interação entre condicionadores de solo + adubação mineral (CON) vs acessos de feijão-fava (AFV) ao nível de $5 \%$ de probabilidade para as variáveis analisadas.

Tabela 1. Análise de variância para número de folhas (NF) e diâmetro do caule (DC) aos 60 dias em função da adubação NPK e uso de condicionadores de solo na cultura do feijão-fava.

\begin{tabular}{cccccccc}
\hline \multicolumn{7}{c}{ Quadrado médio } \\
\hline FV & CON & AFV & CON x AFV & Bloco & Erro & Média & CV (\%) \\
\hline NF & $248,05^{\mathrm{NS}}$ & $0,83^{\mathrm{NS}}$ & $76,20^{\mathrm{NS}}$ & 514,73 & 101,22 & 37,41 & 37,41 \\
DC & $1,73^{*}$ & $1,94^{*}$ & $0,37^{\mathrm{NS}}$ & 1,63 & 0,3 & 5,79 & 9,77 \\
GL & 9 & 1 & 9 & 5 & 95 & - & \\
\hline
\end{tabular}

* = significativo ao nível de 0,05 de probabilidade; NS= não significativo; GL= grau de liberdade; $\mathrm{CV}=$ coeficiente de variação. Fonte: Autores.

Quanto aos acessos 1 e 2 (Tabela 2), pode-se observar que houve diferença significativa quanto ao diâmetro do caule entre os acessos de feijão-fava avaliados, onde o acesso 2 apresentou maior média para diâmetro de caule em relação ao acesso 1. Para os tratamentos com as combinações de NPK\% e condicionadores do solo, as maiores médias para diâmetro do caule (DC) obtidas foram para $\mathrm{N}_{\text {Barvar }}+\mathrm{PK}_{100 \%}, \mathrm{~K}_{\text {Barvar }}+\mathrm{PN}_{100 \%} ; \mathrm{N}_{\text {Barvar }}+\mathrm{K}_{\text {Barvar }}+\mathrm{P}_{\text {Barvar }}+\mathrm{NPK}_{0 \%}$ e $\mathrm{N}_{\text {Barvar }}+\mathrm{K}_{\text {Barvar }}+\mathrm{P}_{\text {Barvar }}+$ $\mathrm{NPK}_{50 \%}$, respectivamente. Uchôa et al. (2020), ao avaliarem o efeito de bioferfilizantes em acessos de feijão-fava, não observaram diferenças entre acessos, apenas para a aplicação de níveis de biofertilização do solo. Para o presente estudo, podese observar que apesar de serem da mesma espécie, os acessos avaliados podem apresentar diferenças entre si, proveniente do potencial genético que cada acesso apresentou nas presentes condições em que a pesquisa foi desenvolvida. Segundo Silva et al. (2015), ao avaliarem a diversidade genética entre acessos de feijão-fava baseado em descritores morfoagronômicos na regiâo Nordeste do Brasil, estes verificaram a presença de variabilidade genética para os acessos avaliados quanto às características quantitativas e qualitativas, destacando-se principalmente quanto ao comportamento vegetativo. 
Tabela 2. Valores médios para diâmetro de caule (DC) aos 60 dias em função da adubação NPK e Uso de condicionadores de solo na cultura do feijão-fava (Phaseolus lunatus L.).

\begin{tabular}{|c|c|}
\hline \multirow{2}{*}{ Tratamento } & Médias \\
\hline & DC (mm) \\
\hline $\mathbf{T}_{1}$ & $5,60 \mathrm{~b}$ \\
\hline $\mathbf{T}_{2}$ & $5,13 c$ \\
\hline $\mathbf{T}_{3}$ & $6,19 a$ \\
\hline $\mathbf{T}_{4}$ & $5,76 b$ \\
\hline $\mathbf{T}_{5}$ & $6,02 \mathrm{a}$ \\
\hline $\mathbf{T}_{6}$ & $5,80 \mathrm{~b}$ \\
\hline $\mathbf{T}_{7}$ & $5,30 \mathrm{c}$ \\
\hline $\mathbf{T}_{8}$ & $5,72 b$ \\
\hline $\mathbf{T}_{9}$ & $6,24 a$ \\
\hline $\mathbf{T}_{10}$ & $6,20 \mathrm{a}$ \\
\hline Média & 5,80 \\
\hline Acesso & DC (mm) \\
\hline $\mathrm{A}_{1}$ & $5,67 b$ \\
\hline $\mathrm{A}_{2}$ & $5,92 \mathrm{a}$ \\
\hline
\end{tabular}

$\mathrm{T}_{1}$ :Controle; $\quad \mathrm{T}_{2}: \mathrm{NPK}_{100 \%} ; \quad \mathrm{T}_{3}: \mathrm{N}_{\mathrm{Barvar}}+\mathrm{PK}_{100 \%} ; \quad \mathrm{T}_{4}: \mathrm{N}_{\mathrm{Barvar}}+\mathrm{PK}_{100 \%}+\mathrm{N}_{50 \%} ; \quad \mathrm{T}_{5}: \mathrm{K}_{\mathrm{Barvar}}+\mathrm{NP}_{100 \%} ;$ $\mathrm{T}_{6}: \mathrm{K}_{\text {Barvar }}+\mathrm{NP}_{100 \%}+\mathrm{K}_{50 \%} ; \quad \mathrm{T}_{7}: \mathrm{P}_{\mathrm{Barvar}}+\mathrm{NK}_{100 \%} ; \quad \mathrm{T}_{8}: \mathrm{P}_{\mathrm{Barvar}}+\mathrm{NK}_{100 \%}+\mathrm{P}_{50 \%} ; \quad \mathrm{T}_{9}: \mathrm{N}_{\text {Barvar }}+\mathrm{P}_{\text {Barvar }}+\mathrm{K}_{\mathrm{Barvar}} ;$ $\mathrm{T}_{10}: \mathrm{N}_{\text {Barvar }}+\mathrm{P}_{\text {Barvar }}+\mathrm{K}_{\mathrm{Barvar}}+\mathrm{NPK}_{50 \%}$. $\mathrm{A}_{1}:$ Luzente; $\mathrm{A}_{2}:$ Venturinha.

Fonte: Autores.

Após sessenta dias da imposição dos tratamentos (Tabela 3), as diferentes combinações de NPK e de condicionadores de solo influenciou o comportamento da cultura do feijão-fava em relação aos parâmetros fisiológicos: concentração intercelular de $\mathrm{CO}_{2}(C i)$, condutância estomática $(g s)$, transpiração $(E)$ e assimilação líquida de $\mathrm{CO}_{2}(A)$. Não observou-se efeito significativo para o fator combinações $(\mathrm{NPK}+\mathrm{CON})$ quanto a eficiência do uso da água (EUA) e eficiência instantânea da carboxilação (EiCi) e para a interação entre as combinações (CON X AFV) e os diferentes acessos de feijão-fava (AFV) para as variáveis referentes as troca gasosas. 
Tabela 3. Análise de variância para concentração interna de $\mathrm{CO}_{2}(\mathrm{Ci})$, condutância estomática (gs), transpiração (E), taxa de assimilação fotossintética (A), eficiência do uso da água (EUA) e eficiência instantânea da carboxilação (EiCi) aos 60 dias em função da adubação NPK e uso de condicionadores de solo na cultura do feijão-fava.

\begin{tabular}{|c|c|c|c|c|c|c|c|}
\hline \multirow{2}{*}{ FV } & \multicolumn{5}{|c|}{ Quadrado Médio } & \multirow{2}{*}{ Média } & \multirow{2}{*}{$\mathrm{CV} \%$} \\
\hline & $\mathrm{CON}$ & AFV & CON X AFV & Bloco & Erro & & \\
\hline$\overline{C_{i}}$ & $1628,44 *$ & $304^{\mathrm{NS}}$ & $766,78^{\mathrm{NS}}$ & 11971,99 & 803,8 & 199,22 & 14,2 \\
\hline$g s$ & $0,001^{*}$ & $0,000003^{\mathrm{NS}}$ & $0,0005^{\mathrm{NS}}$ & 0,002 & 0,0006 & 0,07 & 31,7 \\
\hline$E$ & $0,50 *$ & $0,0003^{\mathrm{NS}}$ & $0,24^{\mathrm{NS}}$ & 0,11 & 0,15 & 1,46 & 26,6 \\
\hline$A$ & $10,05^{*}$ & $4,44^{\mathrm{NS}}$ & $2,58^{\mathrm{NS}}$ & 7,34 & 3,29 & 7,79 & 23,2 \\
\hline $\boldsymbol{E}_{i} \boldsymbol{U} \boldsymbol{A}$ & 1,03 & $0,23^{\mathrm{NS}}$ & $0,55^{\mathrm{NS}}$ & 2,48 & 0,68 & 5,43 & 14,0 \\
\hline$E_{i} C_{i}$ & 0,0003 & $0,000006^{\mathrm{NS}}$ & $0,0001^{\mathrm{NS}}$ & 0,0007 & 0,0001 & 0,04 & 30,9 \\
\hline GL & 9 & 1 & 9 & 5 & 95 & - & - \\
\hline
\end{tabular}

* = significativo ao nível de 0,05 de probabilidade; NS= não significativo; GL= grau de liberdade; $\mathrm{CV}=$ coeficiente de variação.

Fonte: Autores.

Analisando a Tabela 4, nota-se que não houve diferença quanto a concentração interna de $\mathrm{CO}_{2}\left(C_{i}\right)$ entre $\mathrm{T}_{1}, \mathrm{~T}_{2}$ (100\%NPK), $\mathrm{T}_{3}, \mathrm{~T}_{4}, \mathrm{~T}_{9}$ e $\mathrm{T}_{10}$ que receberam o $\mathrm{N}_{\text {Barvar }}$ em suas combinações. Os tratamentos que não receberam $\mathrm{N}_{\mathrm{Barvar}}$ ou reduziu-se a quantidade de fertilizantes recomendados para a cultura do feijão-fava (Trani et. al., 2015), apresentaram as menores médias. Esta variação nos valores médios de concentração interna (Ci) pode está associado aos benefícos do uso do $\mathrm{N}_{\text {Barvar }}$ que pode ter diminuído o estresse hídrico para a cultura, aumentando assim, as trocas gasosas e a fotossíntese (Taiz et. al, 2017). Segundo Jadoski et al. (2005), a redução da concentração interna de $\mathrm{CO}_{2}$ no mesófilo foliar é promovido pelo fechamento dos estomático, reduzindo as trocas gasosas e inibindo a fotossíntese. Segundo Paiva et al. (2005), a taxa de concentração interna de $\mathrm{CO}_{2}$ no mesófilo foliar está associado diretamente com o fechamento dos estômatos e a redução na assimilação de $\mathrm{CO}_{2}$.

Para os resultados obtidos referentes à condutância estomática $\left(\mathrm{g}_{s}\right)$, a média observada foi de $0,079 \mathrm{~mol} \mathrm{CO}_{2} \mathrm{~m}^{-2} \mathrm{~s}^{-1}$ (Tabela 4). Avaliando os efeitos da adubação mineral, orgânica e organomineral na cultura do feijão gurgutuba, Santos (2016) verificou para a condutância estomática $\left(\mathrm{g}_{\mathrm{s}}\right)$ um valor médio de $0,16 \mathrm{~mol} \mathrm{CO}_{2} \mathrm{~m}^{-2} \mathrm{~s}^{-1}$ para as condicões edafoclimáticas de Bananeiras-PB. No presente estudo, a cultura do feijão-fava apresentou uma menor condutância estomática ( $\mathrm{g}_{\mathrm{s}}$ ) quando comparado a cultura do feijão Gurgutuba, sugerindo que a cultura da fava apresenta certa capacidade em resistir à deficiência hídrica. A observação da atividade estomática é fundamental no entendimento dos processos fisiológicos, a qual é o principal meio de trocas gasosas entre a atmosfera e o interior da maquinaria fotossintética (Dutra et. al., 2015). Sendo assim notou-se

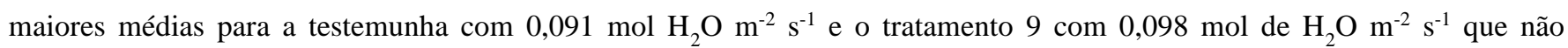
possuem nenhuma porcentagem de adubação mineral, no entanto, o tratamento 9 que recebeu apenas os condicionadores de solo $\left(\mathrm{N}_{\text {Barvar }}+\mathrm{K}_{\text {Barvar }}+\mathrm{P}_{\text {Barvar }}+\mathrm{NPK}_{0 \%}\right.$ ) diferiu significativamente dos demais tratamentos $(\mathrm{p}<0,05)$ que receberam parte da adubação recomanda para a cultura do feijão-fava e/ou condicionadores de solo associados à adubação mineral. Possivelmente, os menores valores de condutância estomática em $\mathrm{T}_{1}, \mathrm{~T}_{2}, \mathrm{~T}_{3}, \mathrm{~T}_{4}, \mathrm{~T}_{5}, \mathrm{~T}_{6}, \mathrm{~T}_{7}, \mathrm{~T}_{8}$ e $\mathrm{T}_{10}$ pode ter sido afetado pelas limitações hídricas conforme observado por Alvino (2012) ao estudar o comportamento fisiológico da cultura do feijão-fava em diferentes regimes de umidade do solo.

As maiores médias referentes a transpiração $(\boldsymbol{E})$ foram as correspondentes a $\mathrm{T}_{1}$ com 1,639 mol de $\mathrm{H}_{2} \mathrm{O} \mathrm{m}^{-2} \mathrm{~s}^{-1}, \mathrm{~T}_{3}$ $\left(\mathrm{N}_{\text {Barvar }}+\mathrm{PK}_{100 \%}\right) \quad$ com 1,508, $\mathrm{T}_{8} \quad\left(\mathrm{P}_{\text {Barvar }}+\mathrm{NK}_{100 \%}+\mathrm{P}_{50 \%}\right)$ e $\mathrm{T}_{9} \quad\left(\mathrm{~N}_{\text {Barvar }}+\mathrm{K}_{\text {Barvar }}+\mathrm{P}_{\mathrm{Barvar}}+\mathrm{NPK}_{0 \%}\right) \quad$ com 1,58 e 1,666 , 
respectivamente. Observando-se que, tais tratamentos ou não receberam a adubação NPK recomendada para a cultura associada ao uso dos condicionadores de solo total ou parcialmente. Essas variações na verdade refletem as consequências de pequenas variações na fisiologia associadas a mudanças na transpiração em condições ambientais diferentes (Taiz et. al., 2017). Mariano (2019), avaliando o comportamento fisiológico da cultura do feijão-fava em função da adubação mineral e orgânica, uso de inoculantes e bioestimulantes observou valores de transpiração variando de 2,067 (Bioestimulante e Adubação mineral) e 2,618 mol de $\mathrm{H}_{2} \mathrm{O} \mathrm{m}^{-2} \mathrm{~s}^{-1}$ (Adubação mineral) em Bananeiras, Estado da Paraíba.

No que se refere ao comportamento da variável fotossíntese líquida (A), a média observada no presente estudo foi de $7,79 \mu \mathrm{mol} \mathrm{CO} \mathrm{m}^{-2} \mathrm{~s}^{-1}$ (Tabela 4). Os maiores valores médios obtidos para fotossíntese líquida (A) foram nos tratamentos que receberam o condicionador de solo $\mathrm{P}_{\mathrm{Barvar}}\left(\mathrm{T}_{7}, \mathrm{~T}_{8}, \mathrm{~T}_{9}\right.$ e $\left.\mathrm{T}_{10}\right)$ e o controle (Tabela 4). Segundo Jacinto et al. (2019), as alterações na fotossíntese líquida podem ser ocasionadas por limitações estomáticas decorrentes das mudanças na abertura dos estômatos e resistência ao influxo de $\mathrm{CO}_{2}$; e devido também às limitações nas reações bioquímicas como a inibição não estomática da fotossíntese em consequência do comprometimento da regeneração da Rubisco. Os mesmos autores, observaram valores médios superiores aos obtidos no presente estudo ao avaliarem as respostas de genótipos de feijão-fava submetidos ao estresse hídrico cultivados em Fortaleza-CE.

Tabela 4. Valores médios de concentração interna de $\mathrm{CO}_{2}(C i)$, condutância estomática $(g s)$, transpiração $(E)$ e fotossíntese (A) aos 60 dias em função da adubação NPK e uso de condicionadores de solo na cultura do feijão-fava.

\begin{tabular}{|c|c|c|c|c|}
\hline & \multicolumn{4}{|c|}{ Médias } \\
\hline & $C i$ & gs & $\boldsymbol{E}$ & $\boldsymbol{A}$ \\
\hline & $\mu \mathrm{mol} \mathrm{m}{ }^{-2} \mathrm{~s}^{-1}$ & $\mathrm{~mol} \mathrm{CO} \mathrm{m}^{-2} \mathrm{~s}^{-1}$ & $\mathrm{~mol} \mathrm{H}_{2} \mathrm{O} \mathrm{m}^{-2} \mathrm{~s}^{-1}$ & $\mu \mathrm{mol} \mathrm{CO}{ }_{2} \mathrm{~m}^{-2} \mathrm{~s}^{-1}$ \\
\hline$T_{1}$ & $206,1 a$ & $0,091 b$ & $1,639 a$ & $8,37 \mathrm{a}$ \\
\hline$T_{2}$ & $210,8 \mathrm{a}$ & $0,079 b$ & $1,455 \mathrm{~b}$ & $7,16 \mathrm{~b}$ \\
\hline$T_{3}$ & $204,1 \mathrm{a}$ & $0,079 b$ & $1,508 \mathrm{a}$ & $7,68 \mathrm{~b}$ \\
\hline$T_{4}$ & $208,6 a$ & $0,078 \mathrm{~b}$ & $1,451 \mathrm{~b}$ & $7,21 \mathrm{~b}$ \\
\hline$T_{5}$ & $194,7 b$ & $0,062 b$ & $1,188 b$ & $6,46 \mathrm{~b}$ \\
\hline$T_{6}$ & $179,2 b$ & $0,055 b$ & $1,096 \mathrm{~b}$ & $6,51 b$ \\
\hline $\boldsymbol{T}_{7}$ & $190,9 b$ & $0,078 b$ & $1,491 \mathrm{~b}$ & $8,34 \mathrm{a}$ \\
\hline$T_{8}$ & $183,1 b$ & $0,084 b$ & $1,580 \mathrm{a}$ & $8,98 \mathrm{a}$ \\
\hline$T_{9}$ & $211,9 a$ & $0,098 \mathrm{a}$ & $1,666 \mathrm{a}$ & $8,84 \mathrm{a}$ \\
\hline$T_{10}$ & $202,8 \mathrm{a}$ & $0,087 \mathrm{~b}$ & $1,475 b$ & $8,32 \mathrm{a}$ \\
\hline Média & 199,22 & 0,079 & 1,455 & 7,79 \\
\hline
\end{tabular}

$\mathrm{T}_{1}:$ Controle; $\mathrm{T}_{2}: \mathrm{NPK}_{100 \%} ; \mathrm{T}_{3}: \mathrm{N}_{\text {Barvar }}+\mathrm{PK}_{100 \%} ; \mathrm{T}_{4}: \mathrm{N}_{\mathrm{Barvar}}+\mathrm{PK}_{100 \%}+\mathrm{N}_{50 \%} ; \mathrm{T}_{5}: \mathrm{K}_{\mathrm{Barvar}}+\mathrm{NP}_{100 \%} ; \mathrm{T}_{6}: \mathrm{K}_{\mathrm{Barvar}}+\mathrm{NP}_{100 \%}+\mathrm{K}_{50 \%} ; \mathrm{T}_{7}: \mathrm{P}_{\mathrm{Barvar}}+\mathrm{NK}_{100 \%} ;$ $\mathrm{T}_{8}: \mathrm{P}_{\text {Barvar }}+\mathrm{NK}_{100 \%}+\mathrm{P}_{50 \%} ; \mathrm{T}_{9}: \mathrm{N}_{\text {Barvar }}+\mathrm{P}_{\text {Barvar }}+\mathrm{K}_{\text {Barvar }} ; \mathrm{T}_{10}: \mathrm{N}_{\text {Barvar }}+\mathrm{P}_{\text {Barvar }}+\mathrm{K}_{\text {Barvar}}+\mathrm{NPK}_{50 \%}$.

Fonte: Autores.

Os tratamentos que não receberam $\mathrm{P}_{\text {Barvar }}$ apresentaram os resultados menos expressivos para a fotossíntese, diferindo-se dos tratamentos que não receberam os condicinadores $\mathrm{N}_{\text {Barvar }}$ e $\mathrm{K}_{\text {Barvar }}+$ proporções de adubação NPK, não diferindo e apresentando valores ligeiramente inferiores ao tratamento controle. 


\section{Considerações Finais}

Os maiores valores obtidos para foram observados nos tratamentos em que se utilizaram os condicionadores de solo e o tratamento controle.

Para as variáveis fisiológicas, de um modo geral, observou-se uma resposta mais eficiente em termos fisiológicos nos tratamentos em que se utilizou os condicionadores de solo via sementes e fertirrigação.

$\mathrm{O}$ uso dos condicionadores do solo promoveram um melhor crescimento inicial e afetou positivamente as trocas gasosas pela cultura do feijão-fava.

\section{Agradecimentos}

Os autores agradecem ao Conselho Nacional de Desenvolvimento Científico e Tecnológico (CNPq), ao Centro de Ciências e Tecnologia Agroalimentar da Universidade Federal de Campina Grande (CCTA/UFCG) e a Green Biotech Brasil pelo auxílio ao presente projeto de pesquisa.

\section{Referências}

Alves, A. U., de Oliveira, A. P., Alves, A. U., Dornelas, C. S., Alves, E. U., Cardoso, E. A., \& Cruz, I. D. S. (2008). Lima beans production and economic revenue as function of organic and mineral fertilization. Horticultura Brasileira, 26(2), 251-254.

Alvino, F. C., Fernades, P. D., Brito, M. E. B., Wanderley, J. A. C., Barreto, C. F., \& Leite, D. T. (2012). Aspectos fisiológicos e produção da cultura da fava sob métodos de captação de água. In: Anais do Workshop Internacional de Inovações Tecnológicas na Irrigação, Fortaleza.

Azevedo, P. (2010). Minhocas, fungos micorrízicos arbusculares e bactérias diazotróficas em mudas de Araucaria angustifolia. 2010. 77f (Doctoral dissertation, Dissertação (Mestrado em Ciências) - Escola Superior de Agricultura “Luiz de Queiroz", Piracicaba).

Barbosa, G. J., \& Arriel, N. H. C. (2018). Feijão-fava e a agricultura familiar de Serraria, PB. Cadernos de Ciência \& Tecnologia, 35(3), 387-403.

Cakmak, I. (2005). The role of potassium in alleviating detrimental effects of abiotic stresses in plants. Journal of Plant Nutrition and Soil Science, 168(4), 521-530.

Canuto, E. D. L., Salles, J. F., Oliveira, A. L. M., Perin, L., Reis, V. M., \& Baldani, J. I. (2003). Resposta de plantas micropropagadas de cana-de-açúcar à inoculação de bactérias diazotróficas endofíticas. Agronomia, 37(2), 67-72.

Cavalcante, R. R., Nascimento, R. I., \& Rocha, C. N. R. (2017). Características produtivas de frutos de abobrinha de moita em função de diferentes doses de adubação nitrogenada. Tecnologia \& Ciência Agropecuária, 11(6), 11-15.

Dutra, A. F., de Melo, A. S., Filgueiras, L. M. B., da Silva, Á. R. F., de Oliveira, I. M., \& Brito, M. E. B. (2015). Parâmetros fisiológicos e componentes de produção de feijão-caupi cultivado sob deficiência hídrica. Revista Brasileira de Ciências Agrárias, 10(2), 189-197.

Fageria, N. K., Barbosa Filho, M. P., \& Stone, L. F. (2003). Resposta do feijoeiro a adubação fosfatada. Informações Agronômicas, Piracicaba, n.102, 1-9.

Ferreira, D. F. (2011). Sisvar: a computer statistical analysis system. Ciência e agrotecnologia, 35(6), 1039-1042.

Fonseca, M. R., Fernandes, A. R., da Silva, G. R., \& Brasil, E. C. (2010). Teor e acúmulo de nutrientes por plantas de feijão caupi em função do fósforo e da saturação por bases. Revista de Ciências Agrárias Amazonian Journal of Agricultural and Environmental Sciences, 53(2), 195-205.

Gyaneshwar, P., James, E. K., Reddy, P. M., \& Ladha, J. K. (2002). Herbaspirillum colonization increases growth and nitrogen accumulation in aluminium-tolerant rice varieties. New Phytologist, 154(1), 131-145.

IBGE (2018) Instituto Brasileiro de Geografia e Estatística. Produção Agrícola Municipal. https://sidra.ibge.gov.br/tabela/1612

Jacinto Júnior, S. G., Moraes, J. G. L., Silva, F. D. B. D., Silva, B. D. N., Sousa, G. G. D., Oliveira, L. L. B. D., \& Mesquita, R. O. (2019). Respostas fisiológicas de genótipos de fava (Phaseolus lunatus L.) submetidas ao estresse hídrico cultivadas no Estado do Ceará. Revista Brasileira de Meteorologia, 34(3), 413-422.

Jadoski, S. O., Klar, A. E., \& Salvador, E. D. (2005). Relações hídricas e fisiológicas em plantas de pimentão ao longo de um dia. Ambiência, 1(1), 11-19.

Mariano, E. D. F. (2019). Atributos químicos do solo, trocas gasosas e produção de fava (Phaseolus lunatus L.) submetida à inoculação e a diferentes fontes de adubação. Dissertação (Mestrado), UFPB - CCHSA, 62f.

Moreira, F. M. S., \& Siqueira, J. O. (2002). Microbiologia e bioquímica do solo. Editora Ufla. p.449-542.

Oliveira, A. P. D., Alves, E. U., Alves, A. U., Dornelas, C. S., Silva, J. A. D., Pôrto, M. L., \& ALves, A. V. (2004). Produção de feijão-fava em função do uso de doses de fósforo. Horticultura Brasileira, 22(3), 543-546. 
Research, Society and Development, v. 10, n. 8, e23510817281, 2021

(CC BY 4.0) | ISSN 2525-3409 | DOI: http://dx.doi.org/10.33448/rsd-v10i8.17281

Oliveira, F. R. A. D., Oliveira, F. D. A. D., Medeiros, J. F. D., Sousa, V. D. F. L. D., \& Freire, A. G. (2010). Interação entre salinidade e fósforo na cultura do rabanete. Revista Ciência Agronômica, 41(4), 519-526.

Oliveira, F. N., Torres, S. B., \& Benedito, C. P. (2011). Caracterização Botânica E Agronômica De Acessos De Feijão-Fava, Em Mossoró, Rn. Revista Caatinga, 24(1), 143-148.

Paiva, A. S., Fernandes, E. J., Rodrigues, T. J., \& Turco, J. E. (2005). Condutância estomática em folhas de feijoeiro submetido a diferentes regimes de irrigação. Engenharia Agrícola, 25(1), 161-169.

Pettigrew, W. T. (2008). Potassium influences on yield and quality production for maize, wheat, soybean and cotton. Physiologia plantarum, 133(4), 670-681.

Römheld, V., \& Kirkby, E. A. (2010). Research on potassium in agriculture: needs and prospects. Plant and soil, 335(1), 155-180.

Santos, H. G., Jacomine, P. K. T., Dos Anjos, L. H. C., De Oliveira, V. A., Lumbreras, J. F., Coelho, M. R., \& Cunha, T. J. F. (2018). Sistema brasileiro de classificação de solos. Brasília, DF: Embrapa, 2018..

Santos, E. M. D. (2016). Produção e qualidade da variedade local de feijão Gurgutuba em resposta a diferentes adubações.

Silva, A. G., Cavalcante, A. C. P., de Oliveira, D. S., \& da Silva, M. J. R. (2015). Crescimento inicial de Phaseolus lunatus L. submetido a diferentes substratos orgânicos e aplicação foliar de urina de vaca. Agropecuária Científica no Semiárido, 11(1), 131-135.

Silva, V. B., Gomes, R. L. F., de Almeida Lopes, A. C., dos Santos Dias, C. T., \& Silva, R. N. O. (2015). Genetic diversity and promising crosses indication in lima bean (Phaseolus lunatus) accessions. Semina: Ciências Agrárias, 36(2), 683-692.

Soil Survey Staff (2014). Keys to soil taxonomy. (12th ed.): USDA, 372p.

Taha, A., Omar, M., \& Khedr, H. (2016). Effect of different sources and levels of potassium on growth, yield and chemical composition of faba bean plants. Journal of Soil Sciences and Agricultural Engineering, 7(3), 243-248.

Trani, P. E., Passos, F. A., Pereira, J. E., \& Semis, J. B. (2015). Calagem e adubação do feijão-vagem, feijão-fava (ou fava-italiana), feijão-de-lima e ervilha torta (ou ervilha-de-vagem). Campinas: IAC.

Troeh, F. R., \& Thompson, L. M. (2007) Solos e fertilidade do solo. Ed. Andrei, 2007.

Taiz, L., Zeiger, E., Møller, I. M., \& Murphy, A. (2017). Fisiologia e desenvolvimento vegetal. Artmed Editora.

Uchôa, M. R., Bastos, R. L. G., de Araújo Viana, T. V., de Azevedo, B. M., de Azevedo, J., Silvestre, F. E. R. S., \& dos Santos, V. C. (2020). Desenvolvimento de cultivares de feijão-fava sob influência de diferentes biofertilizantes. Brazilian Journal of Development, 6(4), 19725-19734.

Zörb, C., Senbayram, M., \& Peiter, E. (2014). Potassium in agriculture-status and perspectives. Journal of plant physiology, 171(9), 656-669. 